\title{
Investigation on harsh environmental effects on polymer fiber optic link for aircraft systems
}

\author{
Sandy Cherian*1, Holger Spangenberg ${ }^{1}$, Reinhard Caspary ${ }^{2}$ \\ ${ }^{1}$ Institut für Flugsystemtechnik, Deutsches Zentrum für Luft- und Raumfahrt e.V. (DLR), Lilienthalplatz 7, \\ 38108 Braunschweig ; ${ }^{2}$ Technische Universität Braunschweig, Institut für Hochfrequenztechnik, Schleinitzstr.22, \\ 38106 Braunschweig, Germany
}

\begin{abstract}
To integrate polymer fiber based physical layer for avionic data network, it is necessary to understand the impact and cause of harsh environments on polymer fiber optic components and harnesses. Since temperature and vibration have a significant influence, we investigate the variation in optical transmittance and monitor the endurance of different types of connector and splices under extreme aircraft environments. Presently, there is no specific aerospace standard for the application of polymer fiber and components in the aircraft data network. Therefore, in the paper we examine and define the thermal cycling and vibration measurement set up and methods to evaluate the performance capability of the physical layer of the data network. Some of the interesting results observed during the measurements are also presented.
\end{abstract}

Keywords: Polymer optical fiber, thermal cycling, vibration measurements

\section{INTRODUCTION}

Fly-by-light systems in aircraft gained immense interest during the last few years as the fiber optic based data transmission in telecommunication and automotive industry matured. A variety of applications in the aerospace industry benefited from the high data transmission capacity together with improved security, immunity to electromagnetic interferences, higher reliability of fiber optic media. However, a stringent requirement of aircraft system data network does not allow the direct mapping of the fiber optic technology from telecommunication and automotive industry. Furthermore, fewer nodes, shorter link lengths and extended performance under harsh environment questions the necessary requirements of optical media for aircraft applications. In order to exploit the full advantage of optical media, we initiated a research on the prospects of integrating polymer optical fiber (POF) instead of silica fiber on aircraft data network. POF with its large core diameter and numerical aperture has acceptable flexibility, relaxed connector tolerance, easy and efficient coupling to high numerical aperture light sources, as well as quick and easy termination procedures.

In order to design a reliable system for avionics data communication it is necessary to understand the impacts of harsh environments on the polymer optic fiber link. We conducted a detailed study on the requirements as a result of the stringent requirements of aircraft systems. The general harsh environmental requirements mainly include the temperature requirements and mechanical requirements. The specific requirements are according to the components used in the data link, path or position of the components, data rate of the link.

The first part of section 2 gives a brief overview of the major deviations to be considered for the design of POF physical layer in comparison to silica fiber based on a baseline study on different military standards $\left[{ }^{1,2,4}\right]$. The section 2.1 and 2.2 examine the detailed harsh environmental requirements and measurement methods on the fiber optic link and its components. The results and discussion part describes the influence of temperature and vibration on fiber optic components and harness.

Photonics Applications for Aviation, Aerospace, Commercial, and Harsh Environments V,

edited by Alex A. Kazemi, et. al. Proc. of SPIE Vol. 9202, 92020I - (c) 2014 SPIE

CCC code: $0277-786 X / 14 / \$ 18 \cdot$ doi: $10.1117 / 12.2061767$

Proc. of SPIE Vol. 9202 92020I-1 


\section{ENVIRONMENTAL REQUIREMENTS}

The requirements for the aircraft systems are different from the telecom/automotive industry. This is mainly due to the difference in the methods to measure, monitor and detect faults and deterioration of performance of the data network. For the aircraft data networks, the link length is in meters rather than in kilometers. Besides, the number of nodes in the aircraft data networks are fewer in comparison to millions of nodes present in telecom networks. Furthermore, aircraft systems are required to operate under extreme operating environments. For example an aircraft system can experience a temperature variation of $-45^{\circ} \mathrm{C}$ to $+85^{\circ} \mathrm{C}$ depending on the area of application. Therefore, integration of polymer optical fiber based physical layer requires a detailed identification of performance and operating requirements and also stable and reliable measurement methods for the acceptance and validation of components and devices.

Initially, we conducted a baseline study on the existing standards and requirements of silica fiber from telecom, automotive and aircraft industry. However, the differences in the material properties and performance characteristics of silica and polymer fiber did not allow the direct adaptation of the requirements. Important parameters to ensure the networks capability to operate according to a design specification are physical properties and optical properties. Physical properties are fiber geometry and material characteristic; optical properties are mainly attenuation, optical cross talk, return loss, numerical aperture, bandwidth, refractive index profile of the fiber. Hence in this section we briefly explain the major performance deviations taken into account for polymer optical fiber in comparison with silica fiber for aircraft applications.

Polymer optical fiber is a step-index multimode fiber which has a core diameter of $980 \mu \mathrm{m}$ and numerical aperture of 0.50 , while silica fiber has a core diameter of $9 \mu \mathrm{m}$ (single mode) and $62.5 \mu \mathrm{m}$ (multimode). In addition, for airborne application the recommended transmission windows of silica fiber are $850 \mathrm{~nm}, 1300 \mathrm{~nm}$, and $1550 \mathrm{~nm}$. These transmission windows are preferred because silica fiber experiences lower attenuation of $0.2 \mathrm{~dB} / \mathrm{km}$ at these wavelengths $\left[^{3}\right]$. However, these recommended transmission windows are not applicable for POF since they have higher attenuation at these wavelengths. POF maintain lower attenuation at $450 \mathrm{~nm}$, $530 \mathrm{~nm}$ and $650 \mathrm{~nm}$. Accordingly, we derived stable attenuation measurement for POF based on MIL-STD-1678 and Telecom standards and conducted the measurements on 6 fiber samples $\left[{ }^{1,3}\right]$. By virtue of these results we agreed to have $650 \mathrm{~nm}$ transmission window for POF applications, which permits the lower attenuation of $0.2 \mathrm{~dB} / \mathrm{m}$ and also the ready availability of matured transmitter technologies. As attenuation of POF significantly varies with regard to the length, the preferred fiber length for data communication is constrained approximately to $100 \mathrm{~m}$. Silica fibers in contrast can operate for $100 \mathrm{~km}$ with lower attenuation. However, for aircraft application short lengths of $50 \mathrm{~m}$ are preferred between the nodes. Furthermore, this large core multimode fiber experiences a considerable variation in power distribution through the modes due to the external disturbances as temperature, vibration, shock etc.

The fiber optic link under consideration for avionic application consists of polymer optical fiber (POF) with high temperature, fiber optic connectors with special terminus for POF, special POF mechanical splices, LED based transmitter with a wavelength of $650 \mathrm{~nm}$, and PIN photo diode as receiver. The deterioration in the performance of other components in the link (such as connectors, splices) are necessary to be analyzed for the calculation of accurate power budget and design of transmission paths with an acceptable loss. Considering the fact that there is no standardized POF connector terminus or mechanical splices, we derived and defined the performance requirements and measurement methods such as attenuation, insertion loss, optical return loss, optical cross talk etc. based on the military standards. 6 samples of each SMA, ST, SC and MIL D38999 connectors, fiber and splices underwent for the measurement of optical characteristion of the components. We followed the general measurement methods as stated in MIL-STD- 1678 with modification according to the properties of each POF component. By considering the numerical aperture, core diameter and number of modes of the fiber the insertion loss of the connector and splices can be in the range of $0.5-1.5 \mathrm{~dB}$ which matches the manufacturer values as well. As stated in the above paragaraph fiber shows an attenuation of $0.2 \mathrm{~dB} / \mathrm{m}$. A cut back method with different launching conditions was used. In order to get a stable measurement meathod we obtained different overfilled launching conditions by lense setup, microscopic setup and setup with mode mixer. The data analysis from each measurement shows for the performance characterisation of fiber it is necessary to include a mode mixer for stable and reliable results. Detailed explanation on the performance requirements and measurement methods of fiber components is not given here as it is out of the scope of this paper. 
The performance and reliable operation of these fiber optic components is certainly influenced by the stringent environmental requirement in aircraft. In this paper, we examined the harsh environmental requirements and measurement methods by considering the performance requirements as a metric for evaluating the endurance of the components at extended operating conditions. These measurement methods are defined by taking account of the special properties of POF, such as the glass transition temperature $\mathrm{T}_{\mathrm{g}}$ of PMMA, large core diameter and the constraints of existing military standards MIL-STD-1687, MIL-STD-1540. Additionally,we investigated the endurance of different connectors such as SMA, SC, ST, MIL D38999 under extended operating conditions since fiber optic connectors and splices have extensive impact on the link loss. In this paper we limit the discussion of harsh environmental measurements to high temperature and vibration measurement definition and methods.

\subsection{High temperature measurements}

This section describes the derivation of thermal cycling measurement method for polymer fiber optic harness by considering its component properties and existing aircraft standards such as MIL-STD-1678 [ $\left.{ }^{1}\right]$. The measurement setup consisted of a light source $(650 \mathrm{~nm})$, photo detector, reference fiber, different types of connectors (SMA, SC, ST and MIL D38999), splices, and temperature chamber. An integrating sphere silicon photo diode with a power meter is used for monitoring the deterioration of optical performance of the components and harness under extreme use conditions. We specifically used this type of detector to get an accurate monitoring regardless of beam shape, angle of incidence and polarization. The photo detector has a wavelength range of $350-1100 \mathrm{~nm}$ and a power range of $1 \mathrm{\mu W}-500 \mathrm{~mW}$. The temperature chamber with a range of $-70{ }^{\circ} \mathrm{C}$ to $+200{ }^{\circ} \mathrm{C}$ is used for the thermal cycling. As we discussed in the earlier section, the length of the fiber was restricted due to attenuation effects. Therefore we modified the standard length of the reference fiber to $13 \mathrm{~m}$ which allowed 3 times cut back method for attenuation measurement.

We realized and maintained a set of initial test conditions throughout the measurements to obtain a stable and reproducible set of measurement results. Firstly, standard ambient conditions of $23 \pm 5{ }^{\circ} \mathrm{C} / 73 \pm 9{ }^{\circ} \mathrm{F}$ and 20 to $70 \%$ RH were realized. Secondly, we carefully avoided all sharp bends, twist and maintained a minimum bend diameter greater than or equal to the minimum long term bend diameter $(20 \mathrm{x}$ cable diameter). Thirdly, we obtained an overfilled launch condition at the source - fiber interface. Finally, we carefully polished and prepared the end face of the fiber.

Prior to thermal cycling, the device under tests (DUT) were tested for attenuation, optical transmittance and insertion loss. By taking account of these optical performance characteristics, the fiber material properties and the aircraft environmental requirements, we modified the span of the temperature profile to $-45^{\circ} \mathrm{C}$ to $+85^{\circ} \mathrm{C}$ for polymer fiber harness $\left[^{1,3,4}\right]$. Thermal cycling was started by placing the prepared DUT in the chamber and maintaining an ambient condition and monitoring the optical transmittance for a duration of 4 hours. This time duration allowed the sample and other measurement devices to achieve a stable condition. In the first step, we ramped down the temperature to a minimum of $-45^{\circ} \mathrm{C}$ and maintained this low temperature for 15 minutes. In the second step, the temperature was ramped up to $+85^{\circ} \mathrm{C}$ and maintained for 15 minutes. Eventually, the temperature profile of the sample was brought back to the standard initial condition. This cycle was repeated 9 times more for each sample. Each sample endured a thermal cycling of approximately 30 hours. Initially, a reference fiber was subjected to the thermal cycling, followed by other DUTs (connectors and splices). This reference fiber serves as the monitor fiber for the insertion loss of other passive components in the link. The connector samples used for measurements were MIL D38999, SMA, ST and SC type. The splices that underwent measurements were splices with and without index matching gel.

\subsection{Vibration measurement setup}

This section explains vibration measurements performed on polymer fiber optic link to demonstrate its operational capability under extended environment. As a baseline profile, we adopted the sine and random vibration input level from MIL-STD-1678 and MIL-STD-1540 shown in Figure 1 and $2\left[^{1,2}\right]$. The DUTs used were fiber, 4 samples of MIL D38999 (with 5 inserts) and mechanical splice. We planned to perform 23 measurements, each with a duration of 30 minutes to evaluate the performance degradation and monitor the endurance of components. A measurement was performed on the first sample for 30 minutes. Since there was no considerable deviation on the power transmission or any signal discontinuity and the limit of the shaker was exceeded, we

Proc. of SPIE Vol. 9202 92020I-3 
reduced the time level from 30 minutes to 3 minutes on each axis of the DUT. We considerd specifically 3 minutes because it is the standard test time of the fiber optic component testing for space applications $\left[{ }^{2}\right]$. Overall, sine and random vibration measurements for the DUT were reduced to a minimum duration of 69 minutes.

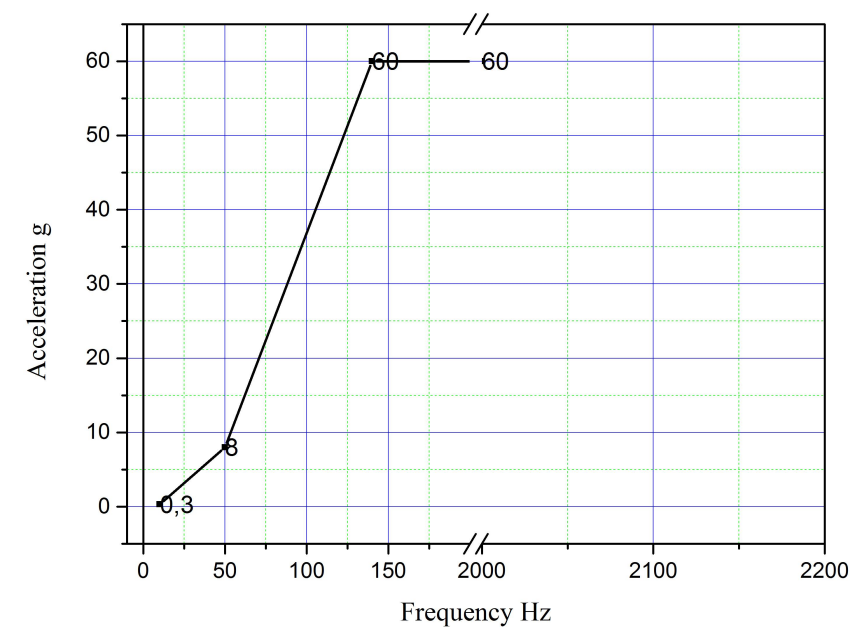

Figure 1. Sine vibration profile according to MIL-STD-1678

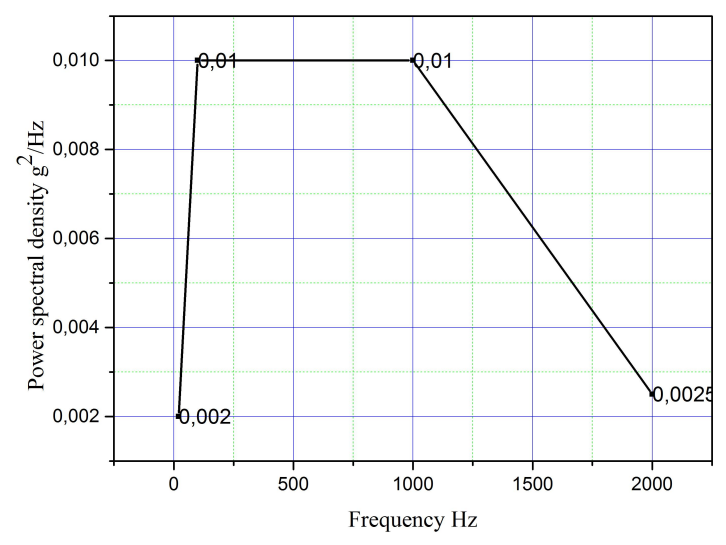

Figure 2. Random vibration profile according to MIL-STD-1540

In accordance with military standards, initially we conducted a vibration resonance search independently on the fixtures and then with the DUT in order to support the design margin or failure evaluation. Eventually, the DUT was mounted on the fixtures and sine and random vibration measurements were performed on three mutually perpendicular axes $\left[{ }^{1,2}\right]$. 


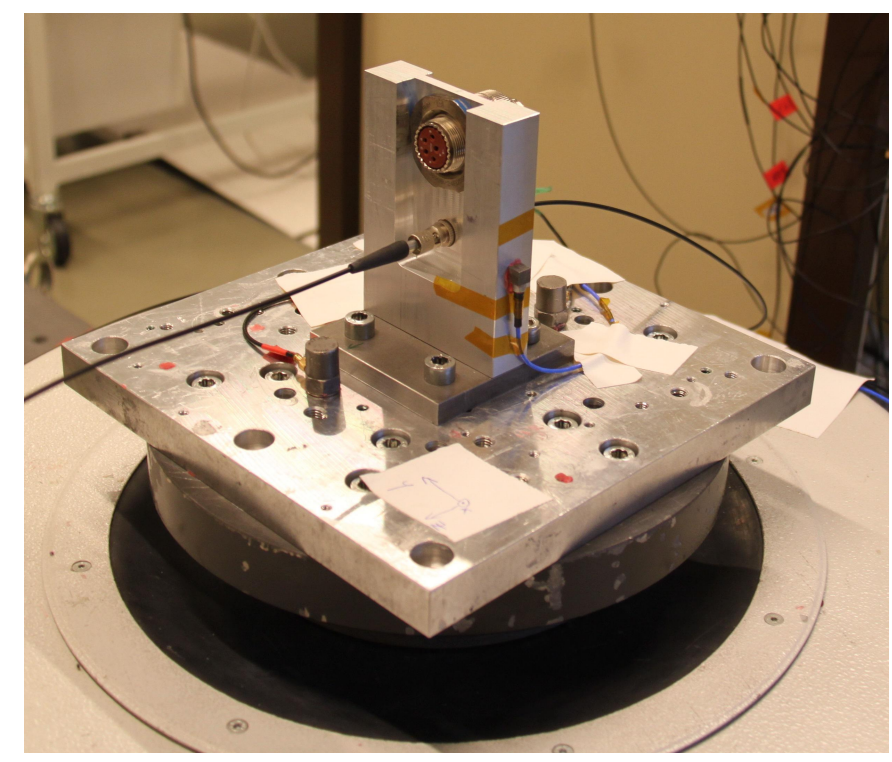

Figure 3. Connector vibration measurement set up in z-axis

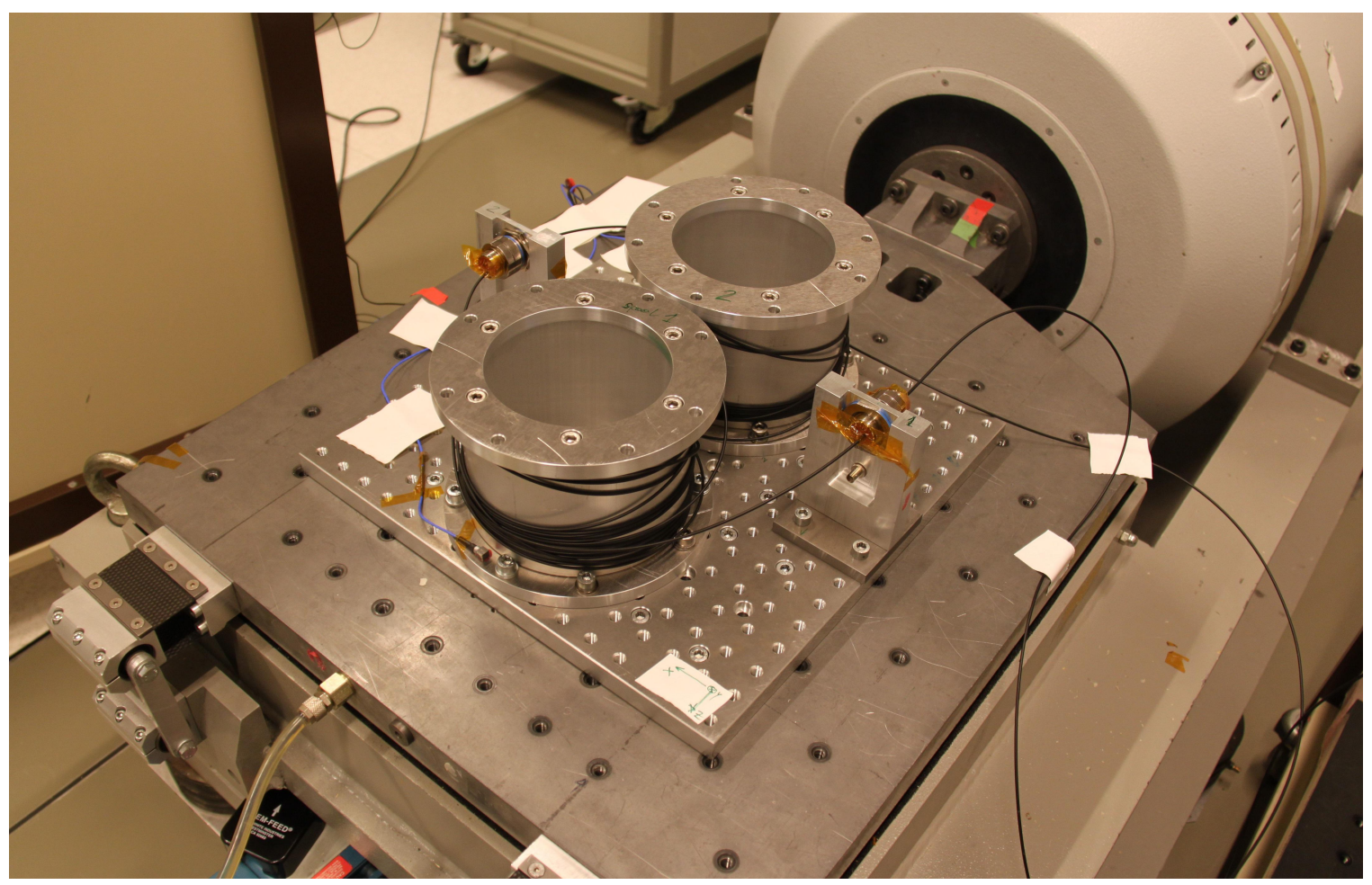

Figure 4. Fiber harness vibration measurement set up in y-axis

Figures 3 and 4 shows the vibration measurement set up of the connectors and whole assembly at the Z-axis and Y-axis respectively. Once the fixture with DUT was mounted on the base plate of the shaker, two controller sensors were connected on the fixture as well as on the base plate and the accelerometer was mounted on the 
DUT to monitor the performance. To achieve Y-axis movement of the DUT, the shaker drum was displaced by 90 degrees.

The optical performance parameter such as change in optical transmittance and optical discontinuity are the components pass/failure criteria for vibration tests. According to MIL-STD-1678 specifications, the maximum allowed change in optical transmittance is $0.5 \mathrm{~dB}$ and change in optical transmittance greater than $0.5 \mathrm{~dB}$ for a duration of more than $50 \mu$ s is considered as optical signal discontinuity $\left[{ }^{1}\right]$. In addition to this, the back-shell of the sample connector shall not show any resonance below $300 \mathrm{~Hz}$ during the vibration test otherwise it is considered as a failure. Verification of resonance was accomplished by monitoring the accelerometer on the backshell of the connector. Prior to and after the random vibration test, a $2 \mathrm{~g}$ sinusoidal search was performed in order to verify the system response. Once the DUT was mounted on the fixture we carried out optical transmittance and insertion loss measurements before the vibration test started. This reference value was recorded and used for the calculation of insertion loss. By considering the limits of the available shaker, the results of the first sample measurement and the weight of the DUT, we modified the vibration input profile as in figure 5 and 6 to the space application standard values $\left.{ }^{2}\right]$.

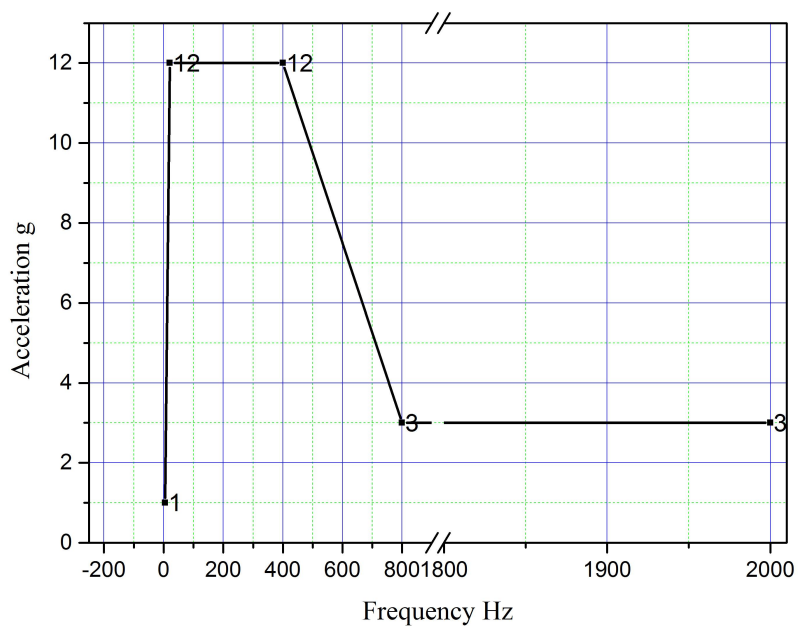

Figure 5. Sine vibration profile used 


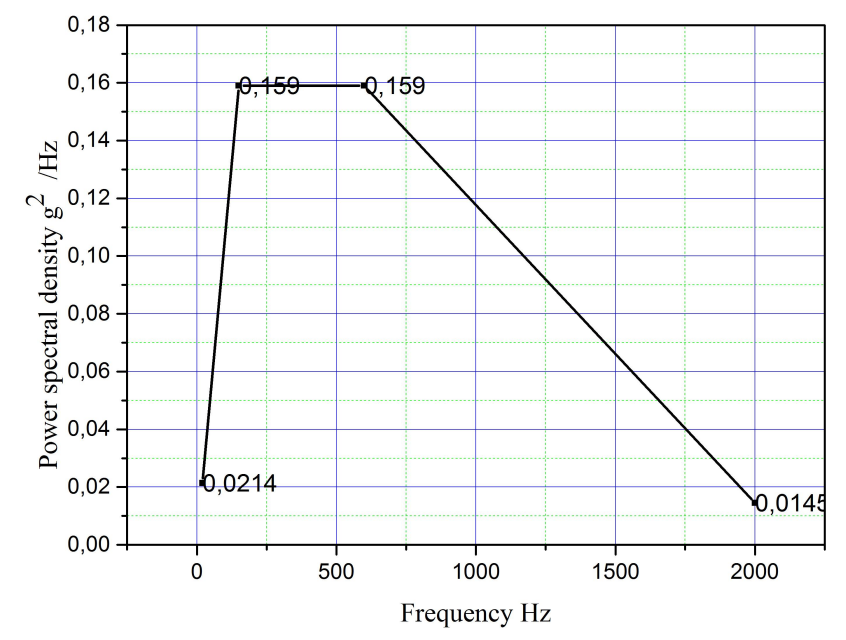

Figure 6. Random vibration profile used

Initial test conditions were maintained as in thermal cycling test. Besides, for each test case, the test set up was arranged in a way to isolate the source, the fiber source interface, the fiber detector interface and the detector from all vibration effects. The fiber was supported flexibly to avoid any unwanted vibration effect during the other component tests. The fixture with DUT was mounted on the base plate of the shaker. Initially vibration profile was implemented on a reference fiber which was then followed by MIL D38999, ST connector and splice.

\section{RESULTS AND DISCUSSION}

We determined the discrepancy in optical transmittance and the endurance of the DUT over extended temperature by analyzing the data collected during the measurement cycle. The change in optical transmittance serves as the metric for components pass/failure criteria for high temperature measurement. In accordance with MIL-STD-1678 specification, maximum allowed change in optical transmittance during thermal cycling is $0.5 \mathrm{~dB}$ which can be evaluated using the equation:

$$
\text { change in optical transmittance }=-\left[10 d B \log _{10}\left(P_{t 1} / P_{m 1}\right)-10 d B \log _{10}\left(P_{t 0} / P_{m 0}\right)\right]
$$

where $P_{m o}$ and $P_{t o}$ are the optical transmittance of reference fiber and DUT measured before the test and $P_{m 1}$ and $P_{t 1}$ are the optical transmittance of reference fiber and DUT measured during the test respectively $\left[{ }^{1}\right]$. Additionally connector end face and splices were visually investigated for any melting or physical damage. Analysis of the data is shown from Figure 7 through 11 which represents the variation in optical transmittance of the different connectors with respect to the temperature and time. 

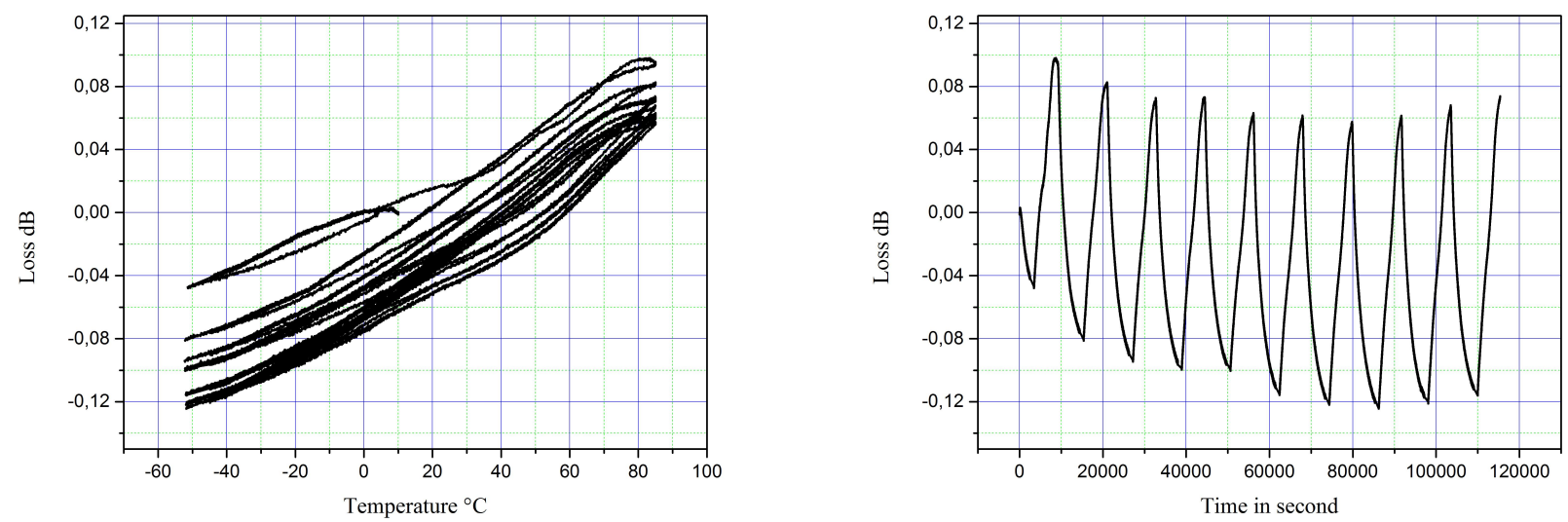

Figure 7. Insertion loss of Reference fiber

Figure 7 represents the effect of temperature on polymer fiber which shows a variation of loss of $0.22 \mathrm{~dB}$ for a duration of 30 hours. These results reveal that polymer fibers with a high temperature resistant jacket can withstand the temperature profile of aircraft systems with an acceptable loss.

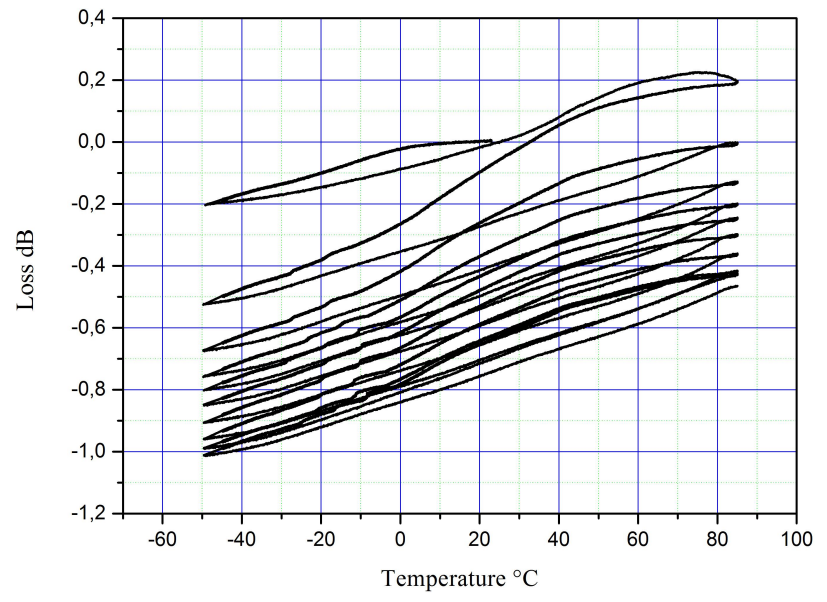

Figure 8. Insertion loss of MIL D38999 connector 


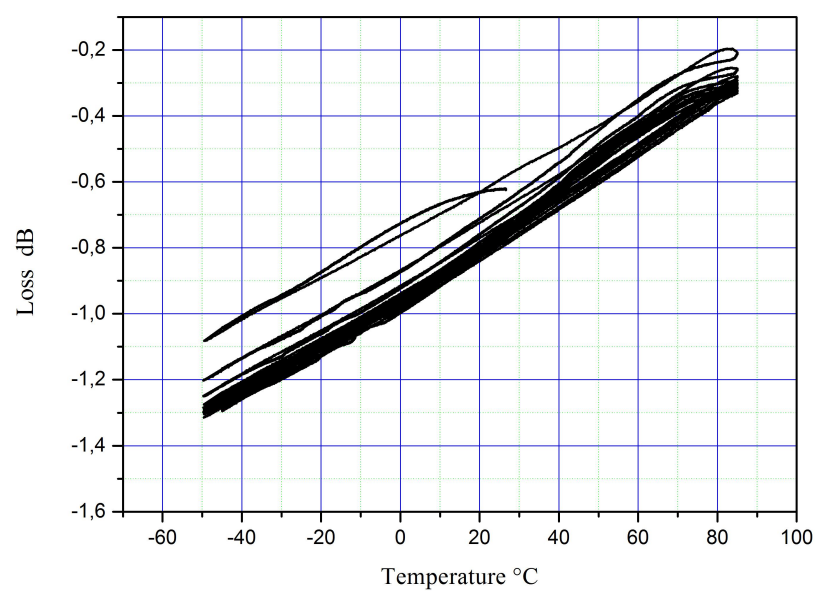

Figure 9. Insertion loss of SC Connector

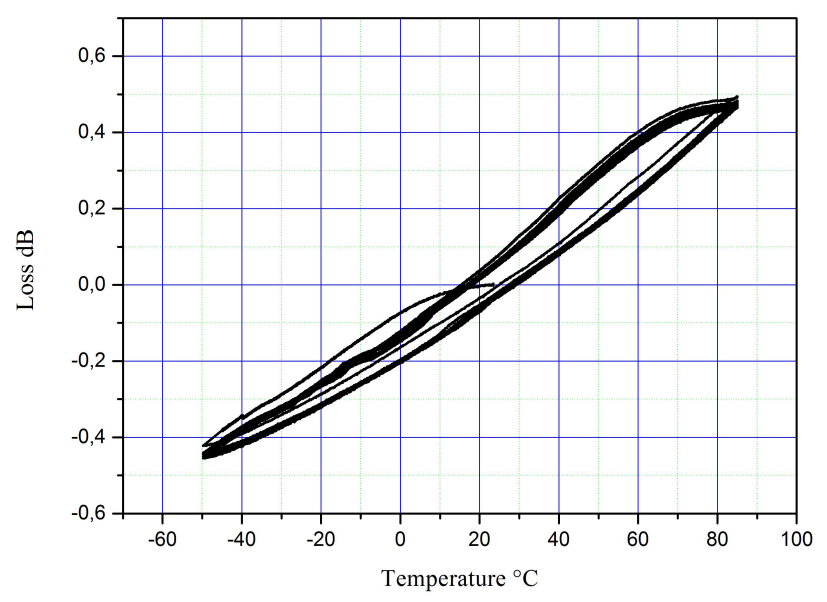

Figure 10. Insertion loss of SMA connector

Proc. of SPIE Vol. $9202920201-9$ 


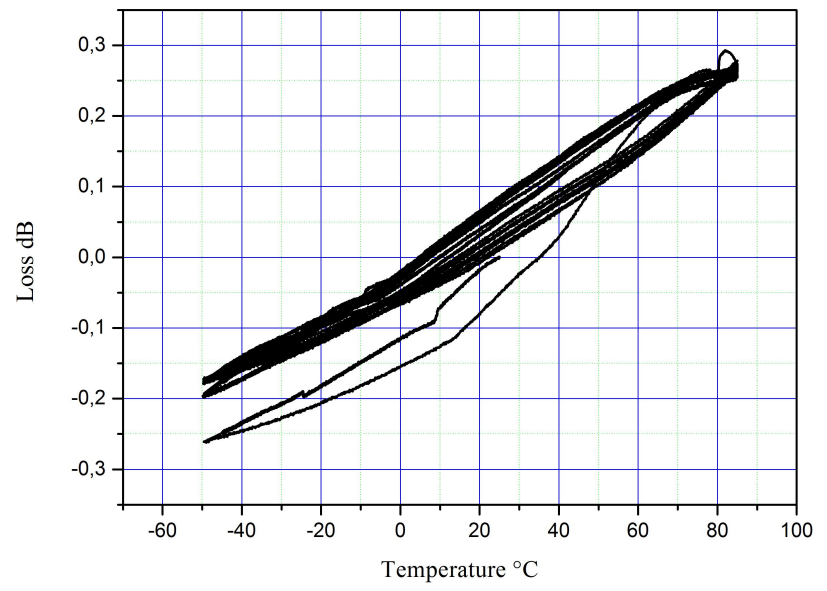

Figure 11. Insertion loss of ST connector

Above graphs from Figures 7 through 11 represent the insertion loss of different types of connectors. All connectors except MIL D38999 show an acceptable insertion loss in the range 0.5 to $1 \mathrm{~dB}$ maximum . The fluctuations between the temperature cycles in MIL D38999 connectors are mainly due to the instability in the clamping of the fiber at the connector fiber interface.

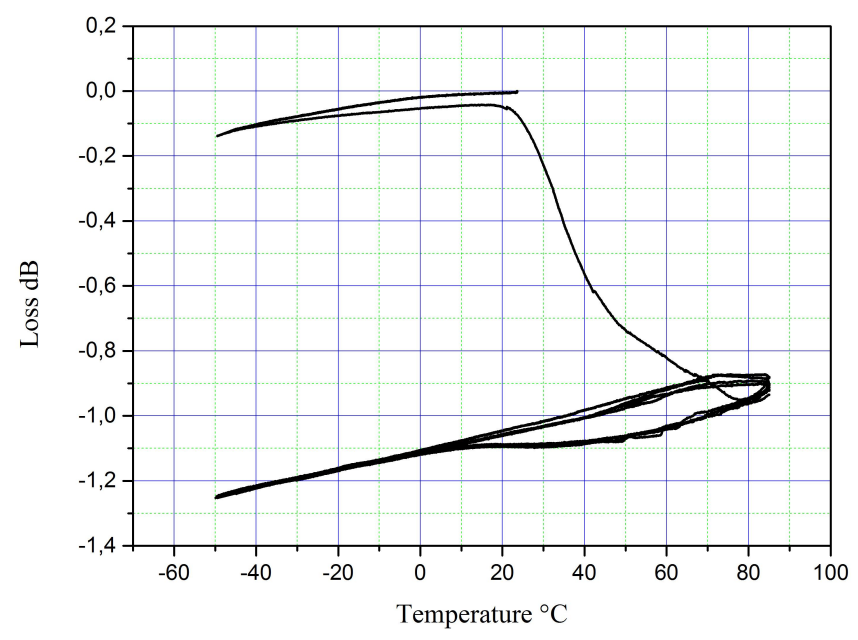

Figure 12. Insertion loss of splice

The result in Figure 12 implies that during the ramp up process in the first cycle the splice has gone through a heat treatment process (annealing). After the first cycle, the splice appears to be very stable in the performance behavior. As a result of the heat treatment on the splice the fiber end faces appeared to have a favorable connection between each other. However, this connection remained stable even at the lower temperatures. We 
used 4 samples to verify the behavior in which all of them showed a similar loss pattern under thermal cycles. Therefore it is advised to use mechanical splices after the annealing process.

We also determined the impact of vibration on the optical transmittance of the fiber optic components and harness. The variation in optical transmittance is calculated by using Eq (1) and optical discontinuity is calculated after each event by

$$
(\Delta D)=10 d B \log _{10}\left(P_{t 1} / P_{t 0}\right)
$$

where $P_{t o}$ is the optical transmittance of DUT measured before the test and $P_{t 1}$ is the optical transmittance of DUT measured during the test $\left[{ }^{1,2}\right]$.

The analyzed data reveals that the vibration affected the insertion loss of the components by $0.02 \mathrm{~dB}$ which is extremely less than the allowed maximum value. The graphs are not shown here since there were no interesting observations made with respect to the insertion loss. Besides, during the test we did not experience any optical signal discontinuity. After the test we examined the fiber,connectors and splice for any physical damage. However, there were no significant visible physical distortion, breakage or displacement on the fiber/connectors/splices or at the fiber connector interface which can induce a signal discontinuity.

\section{CONCLUSION}

High temperature measurement data presented here explain that one can expect an optical performance variation of 0.5 to $2 \mathrm{~dB}$ loss for connectors and splices. However, these values are in the range of manufacturer typical insertion loss values. The connectors showed a stable behavior over the duration of 30 hours of temperature measurements without damaging the fiber. Military connectors experienced slightly higher fluctuations between each temperature cycles than expected manufacturer values. Additionally, vibration measurements did not show any considerable variation in the performance or any signal discontinuity of connectors and splice.

In conclusion, we proposed a stable and reliable test setup and methods for POF, large core fiber connectors and splices. We demonstrated the necessity of modifying the standard requirement value for large core polymer fiber and its link components. In general this work introduces the first step towards the development of standards that enable polymer fiber data networks for aircraft applications.

\section{ACKNOWLEDGMENTS}

We would like to thank the department of Flugversuchstechnik und IT, FT, DLR Braunschweig and Institut für Raumfahrtsysteme, DLR Bremen for helping us in the measurements.

\section{REFERENCES}

1. MIL-STD- 1678, Fiber Optic Cabling System Requirements and Measurements, Department of Defense Standard Practice, 23 July 2009

2. MIL-STD-1540, Test Requirements for Launch Upper Stage and Space Vehicle, Department of Defense Standard Practice, 15 September 1994

3. Peter E Zamzow, Olaf Ziemann,Werner Daum,and Jurgen Krauser, Polymer Optical Fiber for Data Communication, Springer, Berlin, 2002

4. RTCA/DO 160E, Environmental Conditions and Test Procedure for Airborne Equipments, Radio Technical Commission for Aeronautics, 12 September 2004 